For submission to:

Journal of Electroanalytical Chemistry

\title{
Electropolymerization of $\beta$-cyclodextrin onto multi-walled carbon nanotube composite films for enhanced selective detection of uric acid
}

Mulugeta B. Wayu, Luke T. DiPasquale, Margaret A. Schwarzmann, Samuel D. Gillespie, and Michael C. Leopold*

\begin{abstract}
An amperometric uric acid (UA) sensor incorporating a multi-walled carbon nanotubes (MWCNT) network in Nafion and electropolymerized $\beta$-cyclodextrin $(\beta-\mathrm{CD})$ layer is investigated. The electrochemical sensor is comprised of a glassy carbon electrode modified with Nafion-MWCNT nanocomposite film, a $\beta-$ CD polymer inner selective layer, and a Hydrothane polyurethane (HPU) outer selective coating. The surface morphology and electronic structure of the electrode material are characterized using transmission electron microscopy (TEM), scanning electron microscope (SEM), and Fourier transform infrared (FTIR) spectroscopy. The electrocatalytic activity of the sensor is studied using cyclic voltammetry (CV), chronocoulometry (CC) and differential pulse voltammetry (DPV). Analytical performance of the electrochemical sensor scheme with and without MWCNT and/or $\beta-C D$ polymer is determined from direct UA injection during an amperometric analysis. The effective surface area is notably higher for Nafion-MWCNT coated glassy carbon electrodes, which in turn enhanced the sensitivity when coated with $\beta-\mathrm{CD}$ polymer. The results indicated an excellent electrocatalytic property of Nafion-MWCNT/ $\beta-\mathrm{CD}$ film for UA detection with enhanced sensitivity $\left(2.11 \mu \mathrm{A} \cdot \mathrm{mM}^{-1}\right)$, wide linear responses over physiologically relevant concentrations, and fast response times. Enhancement is attributed to MWCNT offering increased electroactive surface area and the ability of $\beta-\mathrm{CD}$ to selectively sequester UA.
\end{abstract}

\footnotetext{
*To whom correspondence should be addressed. Email: mleopold@ richmond.edu. Phone: (804) 2876329. Fax: (804) 287-1897
}

Keywords: electrochemical sensor, amperometric, uric acid, multi-walled carbon nanotubes, layer-by-layer, electropolymerization 


\section{Introduction}

The accurate, reliable, and inexpensive selective detection of uric acid $(2,6,8-$ trihydroxypurine, UA) is clinically significant [1-4]. The presence of abnormal levels of UA in biological fluids such as blood and urine are symptomatic of various diseases such as gout, hyperuricaemia, Lesch-Nyan disease, obesity, diabetes, high cholesterol, high blood pressure, kidney disease and heart disease [5-11]. In addition, the presence of elevated levels of UA in the blood of late term pregnant women indicates higher probability of pregnancy-induced hypertension (PIH). PIH is a condition that can lead to a disorder called pre-eclampsia, which is a significant health risks for both mothers and infants. Emergency cesarean section surgery, a significant abdominal surgical intervention, remain a standard procedure to halt PIH progression even in cases where PIH is only suspected based on hypertension assessment. UA has been identified as a reliable marker to predict PIH and prevent both unnecessary surgeries and preeclampsia cases [12]. Current UA testing procedures require time-consuming laboratory evaluation of blood/urine [7] during which the PIH can remain undiagnosed and increase the probability of serious complications including progression of the condition to pre-eclampsia. Thus, effective UA sensors, capable of accurate, fast, and local monitoring at the bedside, are desired in order to detect and predict abnormal conditions [6, 7, 13].

The co-oxidation of interferences at common modified electrodes used as UA sensor interfaces augments the problem of selective detection of the analyte [1,3]. To overcome these obstacles, enzymes have been incorporated at the interface of modified electrodes for use as biosensors $[1,13,14]$. These enzyme-based biosensors show a high degree of selectivity in the detection of specific small molecules. However, the use of enzymes invokes indirect measurements of target analyte concentration, are often dependent on enzyme kinetics and/or availability, and can have low sensitivity [4]. First generation biosensors, for instance, employ enzymatic reactions where an immobilized oxidase enzyme in the presence of oxygen catalyzes a specific analyte and yields hydrogen peroxide $\left(\mathrm{H}_{2} \mathrm{O}_{2}\right)$ byproduct. In most first generation schemes, the $\mathrm{H}_{2} \mathrm{O}_{2}$ is then oxidized at the working electrode to yield a current signal proportional to the reacted analyte concentration [13-16]. Furthermore, enzyme-based biosensors have shown many disadvantages such as poor stability and reproducibility, oxygen dependence, high costs, and lengthy preparation times $[2,17]$. 
Due to their fascinating chemical, electronic, mechanical and structural properties, nanomaterials (NMs) have been used at modified electrode sensing schemes as a strategy to provide enhanced sensitivity, including a number of studies using metallic nanoparticles [18-21]. Another aspect of this approach for effective sensors has involved the incorporation of carbonbased NMs, including recent work with graphene for the detection of UA[22, 23]. Carbon nanotubes (CNT), for example, have been integrated into many devices including electrochemical sensors $[16,24]$. CNT behave as metallic or semiconductor materials based on their atomic structure. When used to modify a working electrode, CNT have shown the ability to promote electron-transfer in chemical reactions [10, 16, 25-29]. Multi-walled carbon nanotubes (MWCNT) are considered to be particularly effective electrode supplement materials due to their large surface areas, high conductivities, and demonstrated electrocatalytic activities which allow fast electron-transfer and ability to reduce required over-potentials [30, 31]. However, due to their strong inter-tube van der Waals forces of attraction, the insolubility of CNT in aqueous or organic solvents remains problematic [10]. Numerous methods have been adopted to modify CNT in order to enhance its dispersion in solvents [10, 32]. Nafion, a perfluorosulfonate ionomer, has been applied for charge exclusion [33], increased matrix chemical/mechanical stability [34] and is known to have high water permeability with no significant swelling upon immersion in aqueous solutions [27, 34-37]. When used as a polymeric modification of an electrode, Nafion offers stable and homogeneous thin films [37, 38]. In addition, Nafion-ethanol solutions have been used to homogeneously suspend CNT [27].

Direct electrochemical detection of UA using non-enzymatic strategies are gaining attention and generally focus on UA oxidation at modified electrodes as the signal:

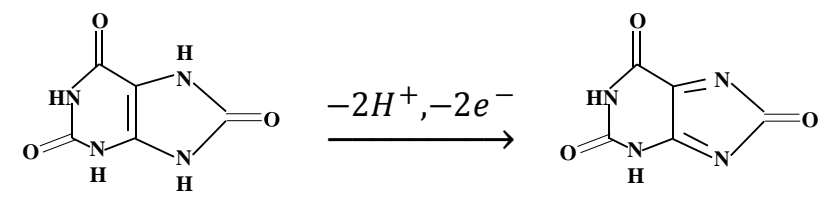

This strategy presents the inherent challenge of introducing effective selectivity without employing an enzymatic reaction [4, 39, 40]. Cyclodextrins (CD) are a group of naturally occurring macrocyclic glucose oligomers that form basket-like structures known as $\alpha, \beta$ and $\gamma-$ 
CD with six, seven and eight D-glucose units, respectively. The $\alpha, \beta$ and $\gamma-C D$ structures create molecular cavities of $0.53,0.65$, and $0.83 \mathrm{~nm}$ in diameter, respectively [9, 27, 40]. CD consists of primary and secondary hydroxyl groups on the smaller and larger edges or rims, respectively, as well. In addition to the hydrophilic regions at the edges, $\mathrm{CD}$ composed of $\mathrm{C}-\mathrm{H}$ bonds point towards the inside of the cavity, which render hydrophobic character to the interior region [41]. The combination of these functional groups in their molecular structure, enables the CD to form supramolecular complexes. For instance, in the cavity structure, hydrophobic molecules can easily be incorporated by displacing the water [28, 42, 43]. More specifically, for example, $\beta-$ CD can form a host-guest inclusion complex with UA via hydrogen-bonding and hydrophobic interactions $[2,3]$. It has been reported that immobilized CD onto electrode surfaces enhanced the selective detection of UA in the presence of ascorbic acid, a common interferent species [9, 44].

Herein, two materials are evaluated in terms of their ability to enhance the performance of a non-enzymatic, layer-by-layer (LbL)-developed UA electrochemical sensor: the incorporation of MWCNT network within a Nafion matrix and the electropolymerization of $\beta-C D$ onto the Nafion-MWCNT nanocomposite modified electrodes. To the best of our knowledge, the synergistic effect of the electropolymerization of $\beta-\mathrm{CD}$ in conjunction with Nafion-MWCNT network for selective and sensitive amperometric detection of UA has not yet been explored. Our research includes extensive characterization of the structure and morphology of the electrode materials using transmission electron microscopy (TEM), scanning electron microscope (SEM), atomic force microscopy (AFM) and Fourier transform infrared (FTIR) spectroscopy. The electrochemical behavior and the electrocatalytic properties of the electrode materials were investigated using cyclic voltammetry $(\mathrm{CV})$, chronocoulometry $(\mathrm{CC})$, chronoamperometry $(\mathrm{CA})$ and differential pulse voltammetry (DPV).

\section{Experimental Section}

\subsection{Materials and Instrumentation.}


All chemicals were purchased from Sigma-Aldrich unless specifically stated. Hydrothane AL25-80A polyurethane (HPU) was obtained from AdvanSource Biomaterials. Nafion (Liquion solution LQ-1105 1100EW 5\% wt.) purchased from Ion Power, Inc. (New Castle, DE, USA). Ultra-purified water $\left(\mathrm{UP} \mathrm{H}_{2} \mathrm{O}, 18.3 \mathrm{M} \Omega \cdot \mathrm{cm}\right)$ was used to prepare all solutions. An eight-channel potentiostat (CH Instruments, 1000B) was used to record amperometric current-time (I-t) curves to evaluate the analytical performance of the sensors, as described below. Electrochemical cells were comprised of a common $\mathrm{Ag} / \mathrm{AgCl}$ (saturated $\mathrm{KCl}$ ) reference electrode, a common platinum wire counter electrode (Sigma-Aldrich), and modified glassy carbon working electrodes (3 mm diameter, CH Instruments).

\subsection{Electrode Preparation}

Glassy carbon electrodes (GCEs) were successively polished with 1.0, 0.3, and $0.05 \mu \mathrm{m}$ alumina powder (Electron Microscopy Sciences) and rinsed thoroughly with ultrapure water (UP $\mathrm{H}_{2} \mathrm{O}$ ) prior to use. The pristine multi-walled carbon nanotube-Nafion (Nafion-MWCNT) nanocomposite was prepared as follows. First, $2.0 \mathrm{mg}$ of pristine MWCNT were dispersed in 1.0 $\mathrm{mL}$ of $0.5 \%$ wt Nafion solution in ethanol using 30 min. sonication. Second, $10.0 \mu \mathrm{L}$ of NafionMWCNT nanocomposite solution was drop casted onto the mirror finished GCE. The electrode was then dried at room temperature for $30 \mathrm{~min}$.

Electropolymerization of CD on bare GCE or Nafion-MWCNT nanocomposite modified GCE was carried out by cyclic voltammetry (CV) in $0.01 \mathrm{M} \mathrm{CD}$ in PBS ( $\mathrm{pH}=7.00)$. The bare GCE or GCE/Nafion-MWCNT working electrode was immersed in this solution and the potential was scanned from -0.8 to $1.30 \mathrm{~V}$ versus $\mathrm{Ag} / \mathrm{AgCl}$ reference electrode during successive cycles at $0.10 \mathrm{Vs}^{-1}$ scan rate. After the $\mathrm{CD}$ polymer was generated onto bare GCE or GCE/Nafion-MWCNT electrode, the GCE/CD and GCE/Nafion-MWCNT/CD electrodes formed was dried at room temperature for $30 \mathrm{~min}$.

The outer polyurethane (PU) layer semipermeable membrane was applied as previously reported [14, 20, 21, 45]. Briefly, $100 \mathrm{mg}$ of Hydrothane polyurethane (HPU) was dissolved in ethanol and THF mixture (1:1) ratio, which was stirred overnight. A $10.0 \mu \mathrm{L}$ aliquot of the HPU 
blend was then deposited on the Nafion-MWCNT/CD modified electrode and allowed to dry (ambient, $30 \mathrm{~min}$.) to obtain GCE/Nafion-MWCNT/CD/HPU electrochemical sensor. For the purpose of comparison, various electrodes such as GCE/CD/HPU, GCE/Nafion-MWCNT/HPU were prepared in similar fashion.

\subsection{Film Characterization and Sensor Performance Evaluation}

Chronocoulometry (CC), the measurement of charge (coulombs) as a function of time (chrono) [46], was employed as previously described [15] using the slope of Anson plots and equation 1:

$$
Q=2 n F A C D^{1 / 2} \pi^{-1 / 2} t^{1 / 2}
$$

where $\mathrm{Q}$ is the charge passed (C), $\mathrm{n}$ is the number of electrons transferred, $\mathrm{F}$ is Faraday's constant $(96,500 \mathrm{C} /$ mole $), \mathrm{A}$ is the electroactive surface area $\left(\mathrm{cm}^{2}\right), \mathrm{C}$ is $\left[\mathrm{Fe}(\mathrm{CN})_{6}\right]^{3-/ 4-}$ concentration $(5.00 \mathrm{mM}$ in $0.50 \mathrm{M} \mathrm{KCl}), \mathrm{D}$ is the diffusion coefficient for $\left[\mathrm{Fe}(\mathrm{CN})_{6}\right]^{3-/ 4-}(7.6$ $\mathrm{x} 10^{-6} \mathrm{~cm}^{2} / \mathrm{s}$ ), and $\mathrm{t}$ is the time (s). The electroactive surface area was calculated from the slope of Anson plots (Supplementary Material). The fabricated electrochemical sensors were placed in $65.55 \mathrm{mM}$ potassium phosphate buffer solution $(\mathrm{pH}=7.0)$, to simulate near-physiological $\mathrm{pH}$ and ionic strength [13]. To stabilize the sensor reading, all biosensors were subjected to $+0.30 \mathrm{~V}$ vs $\mathrm{Ag} / \mathrm{AgCl}$ in $25 \mathrm{~mL}$ of PBS for 20 minutes. During testing, $50 \mu \mathrm{L}$ aliquots of $50 \mathrm{mM}$ UA stock solution were injected at $200 \mathrm{~s}$ intervals while stirring $(1100 \mathrm{rpm})$ to obtain stair-step response to successive $100 \mu \mathrm{M}$ UA increases. As in prior work, slopes of calibration curves (i.e., current response vs. UA concentration) corresponded to sensitivity while response times $\left(t_{R-95 \%}\right)$ were defined as the time required to reach $95 \%$ of the total change in current due to an increase in UA concentration [20, 21]. Instrumentation details and procedures for microscopy (AFM, TEM, and SEM) and spectroscopic (FTIR) characterization of the films is included in Supplementary Material. 


\section{Results and Discussion}

The overall design of the non-enzymatic layer-by-layer (LbL) UA electrochemical sensor is depicted in Scheme $\mathbf{1}$ and features three major components modifying a glassy carbon electrode: (1) Nafion-MWCNT nanocomposite layer; (2) a cyclodextrin electropolymerized layer and (3) an outer-selective polyurethane layer. As shown in Scheme1, MWCNT was dispersed in Nafion using sonication to form Nafion-MWCNT nanocomposite solution. The Nafion-MWCNT nanocomposite solution was then drop casted onto mirror finished GCE to obtain GCE/Nafion-MWCNT. Then cyclodextrin was electropolymerized onto GCE/NafionMWCNT to obtain GCE/Nafion-MWCNT/CD. Finally, HPU blend was deposited onto GCE/Nafion-MWCNT/CD and allowed to dry (ambient, $30 \mathrm{~min}$.) to obtain GCE/NafionMWCNT/CD/HPU. In general, the use of these components as a composite film was shown to function over extended linear/dynamic ranges of detection with high sensitivity, fast response and selectivity.

\subsection{Nafion-MWCNT Composite Film Electrode Modification}

The cyclic voltammetry (CV) behavior of potassium ferricyanide $\left(\left[\mathrm{Fe}(\mathrm{CN})_{6}\right]^{3-/ 4-}\right)$ remains an effective method to monitor the surface properties of modified electrodes, since electron transfer occurs either by tunneling through an interface or at defects in the interfacial barrier between the solution species and the electrode $[47,48]$. In this study, the $\left[\mathrm{Fe}(\mathrm{CN})_{6}\right]^{3-/ 4-}$ probe was chosen to examine changes of electrode surface characteristics upon modification by Nafion or MWCNT material. Figure 1A shows typical CVs of $\left[\mathrm{Fe}(\mathrm{CN})_{6}\right]^{3-/ 4-}$ observed at bare GCE, Nafion modified GCE, and Nafion-MWCNT nanocomposite films modified GCE. As expected, well-defined oxidation and reduction peaks are observed at the unmodified or bare GCE. Upon modification with Nafion, the peak current is significantly attenuated and the diffusional peak shape diminished compared to the bare electrode due to the $\left.\mathrm{Fe}(\mathrm{CN})_{6}\right]^{3-/ 4-}$ probe being blocked/hindered (Fig. 1A, b). The GCE/Nafion-MWCNT modified electrode, however, exhibited peak current comparatively higher to that of the bare electrode, illustrating that the MWCNT modified electrode possesses higher electrocatalytic activity resulting from increased 
electroactive surface area. Furthermore, the peak-to-peak potential separation between the cathodic and anodic peaks of the GCE/Nafion-MWCNT is slightly smaller $\left(\Delta \mathrm{E}_{\mathrm{p}}=128 \mathrm{mV}\right)$ than that for the bare GCE $\left(\Delta \mathrm{E}_{\mathrm{p}}=197 \mathrm{mV}\right)$ suggesting that the MWCNT are facilitating faster electron transfer kinetics [49]. It should be noted, the enhanced current response obtained with MWCNT incorporation was evident regardless of how the voltammetry peaks were analyzed (i.e., isolating Faradaic current manually or background subtracted voltammetry). Additionally, differential pulse voltammetry (DPV), a technique that can more effectively discriminate against background charging current, reinforced the CV results, showing Nafion modification as a barrier to $\left[\mathrm{Fe}(\mathrm{CN})_{6}\right]^{3-/ 4-}$ and the introduction of MWCNT to the Nafion as enhancing the redox probe signal (Supplementary Material, Figure SI-1A-B and Table SI-1), consistent with literature [49].

The electroactive surface areas of the bare GCE, Nafion and Nafion-MWCNT nanocomposite modified GCE were examined using chronocoulometry (CC) in $5.0 \mathrm{mM}$ $\left[\mathrm{Fe}(\mathrm{CN})_{6}\right]^{3-/ 4-}$ and $0.5 \mathrm{M} \mathrm{KCl}$ solution as well. $\mathrm{CC}$ of $\left[\mathrm{Fe}(\mathrm{CN})_{6}\right]^{3-/ 4-}$ at the three interfaces are shown in the Supplementary Material (Figure SI-1C). CV and DPV results showed enhanced current response $(\sim 1.7 \mathrm{X})$ for the redox reaction of $\left[\mathrm{Fe}(\mathrm{CN})_{6}\right]^{3-/ 4-}$ on the bare GCE vs NafionMWCNT nanocomposite modified GCE. This significant current response increase is due to the apparent two-fold increase in electroactive surface area that is achieved with the use of the Nafion-MWCNT nanocomposite coatings (Table SI-1). MWCNT are known for large surface area [50] and the results suggest that they are effectively coupled to the electrode interface within the Nafion matrix. A summary of all CC, CV and DPV results are included in the Supplementary Material (Table SI-1).

Electrodes were modified with Nafion-MWCNT nanocomposite films and subsequently covered with an outer selective hydrophilic polyurethane layer for the direct electrochemical detection of UA. The outer selective PU, specifically a layer of $100 \%$ Hydrothane (HPU), has been shown in previous work to be selective for UA [13]. That particular work [13] established several important properties of a 100\% HPU layer used as a selective layer for UA sensing, including specific permeability toward UA with corresponding less permeability for certain specific interferents [13]. Since UA is electroactive, cyclic voltammetry of $1 \mathrm{mM}$ UA in PBS at various modified electrodes, including bare and Nafion-MWCNT modified GCE, each with 
HPU coatings, showed the presence of a strong UA oxidation peak $(-7.23 \mu \mathrm{A})$ at $+0.3 \mathrm{~V}$ with both peak shape and potential consistent with prior work [13]. Representative examples of the observed CV are included in Supplementary Material (Figure SI-2) and also illustrate the notable increase in background charging current from systems doped with MWCNT and their increased surface area. Based on the peak potential of this UA oxidation, electrodes to be tested as UA sensors were held at $+0.3 \mathrm{~V}$ during chronoamperometry to generate current-time (I-t) curves during standardized injections of UA.

Figure 1B, shows the chronoamperometric response of UA at the two modified electrodes: bare GCE with HPU and GCE modified with Nafion-MWCNT nanocomposite film capped with HPU. Notably, there is a near-negligible current response recorded for the GCE/HPU while a well-defined, stair-step I-t response is observed at the Nafion-MWCNT nanocomposite film modified GCE, noted as GCE/Nafion-MWCNT/HPU. The I-t responses translates into a calibration curve for UA that is linear across the physiologically relevant range of UA $(0.2-0.5 \mathrm{mM})$ [1]. The composite showed substantially enhanced sensitivity $(1.48 \pm 0.20$ $\left.\mu \mathrm{A} \cdot \mathrm{mM}^{-1}\right)$, which is higher than reported using uricase enzymes $[8,13]$. The poor $\mathrm{I}-\mathrm{t}$ response at the GCE/HPU and its corresponding calibration curve $\left(0.02 \pm 0.003 \mu \mathrm{A} \cdot \mathrm{mM}^{-1}\right)$ are included as isolated figures in Supplementary Material (Figure SI-3) for comparison. Thus, even though high sensitivity toward UA is achieved with the Nafion-MWCNT composite modification, other than the semi-permeable HPU, the system lacks sufficient selectivity to discriminate against common interferents (Figure SI-9).

\subsection{Surface characterization of Nafion-MWCNT Nanocomposite} Films

The surface morphologies and structures of the electrode materials as well as the influence of the supramolecular environment were studied using atomic force microscopy (AFM), transmission electron microscopy (TEM), scanning electron microscopy (SEM), and Fourier transform infrared (FTIR) spectroscopy. AFM imaging of pristine MWCNT, examples shown in Supplementary Material (Figure SI-4), are typical of this material [51]. TEM images of 
pristine MWCNT used in the aforementioned sensing scheme are presented in Figure 2a with additional examples included in Supplementary Material (Figure SI-5). These images are also typical of MWCNT and include instances of exposed inner concentric cylinders (Figure 2a, black arrows), characteristic of the multi-walled nature of these materials. When pristine MWCNT material is dispersed in $0.5 \%$ wt Nafion, TEM imaging captures the clear presence of the fibrous MWCNT homogeneously distributed throughout the film (Supplementary Material, Figure SI5). Nafion is known to aid in the uniform dispersion of MWCNT [18, 38]. TEM imaging of the Nafion-MWCNT composite film (Figure 2c) reveals MWCNT structure that has been substantially modified compared to the pristine MWCNT in that there is a substantial increase in average diameter and the appearance of helical morphology around the tube structure - Nafion wrapping around the MWCNT (Fig. 2c, white arrow). It is established that the exceptional rigidity, cylindrical shape, and perfect straightness of CNT render favorable conditions for the growth of helical crystals of proteins [38] and it is suspected a similar phenomenon is taking place with the Nafion polymer. Similarly, SEM imaging, while not as detailed as the TEM visualization, shows a difference between the pristine MWCNT material and the NafionMWCNT composite, Fig. 2b and 2d, respectively. A similar polymeric structure (Fig. 2d, white arrow) is observable throughout the Nafion-MWCNT images that is notably absent from images of only agglomerated MWCNT (Fig. 2b and 2d, black arrows), a characterization consistent with established literature [45]. The TEM and SEM images of a LbL full composite film (i.e.; NafionMWCNT with electropolymerized $\beta-\mathrm{CD}$ ) are shown in Figures $2 \mathrm{e}$ and $2 \mathrm{f}$, respectively, suggest the successful immobilization of $\beta-\mathrm{CD}$ (dotted black arrows) onto GCE/Nafion-MWCNT via electropolymerization [52].

Infrared spectroscopy was employed as a supplemental spectroscopic characterization of the MWCNT-doped Nafion films. Figure 3 shows characteristic FTIR spectra of both Nafion and MWCNT. The vibrational bands of Nafion were assigned based on the previously published data [53-55] where peaks at 1220, 1150, 1060 and $980 \mathrm{~cm}^{-1}$ are attributed to $\mathrm{CF}_{2}$ broad asymmetric stretching, $\mathrm{CF}_{2}$ asymmetric stretching, $\mathrm{S}-\mathrm{O}$ asymmetric stretching and $\mathrm{C}-\mathrm{F}$ stretching of the $-\mathrm{CF}_{2}-\mathrm{CF}\left(\mathrm{CF}_{3}\right)$ group, respectively - a spectroscopic signature for the material [55]. Similarly, pristine MWCNT material yields a notably featureless spectrum that has been previously observed as well [56]. Figure 3 also shows the IR spectra of the Nafion-MWCNT 
composite where features of both spectra from the neat materials are retained, including signature peaks of Nafion at 1220 and $980 \mathrm{~cm}^{-1}$. The IR spectroscopy provides additional evidence to the successful preparation of Nafion-MWCNT nanocomposite, consistent with TEM and SEM results (Fig. 2).

\subsection{Electropolymerization of $\beta-C y c l o d e x t r i n$ for Selectivity}

Whereas enzymatic-based sensors provide natural selectivity, non-enzymatic sensors necessitate adding selectivity via other means. Cyclodextrin-based electrodes have been previously employed to facilitate amperometric and voltammetric UA sensors with enhanced sensitivity and selectivity $[2,3,44]$. Here, the natural cavity size of $\beta-C D$ cylinder is targeted to allow for the selective sequester of UA via the displacement of water and internal intermolecular interaction [2]. Electropolymerization of $\beta-\mathrm{CD}$ using cyclic voltammetry is employed to modify electrodes with a polymer layer capable of providing UA selectivity. Examples of the $\beta-C D$ electropolymerization voltammetry are provided in Supporting Material (Figure SI-6). As in prior voltammetry at these modified electrodes, a significant increase in current $(\sim 20$ fold $)$ is observed at Nafion-MWCNT modified electrodes. The successful electropolymerization of $\beta-$ $\mathrm{CD}$ is also confirmed using $\left[\mathrm{Fe}(\mathrm{CN})_{6}\right]^{3-/ 4-}$ (Figure SI-9).

Figure 4 shows chronoamperometry of standard UA injections at a GCE modified via electropolymerization of $\beta-\mathrm{CD}$ via cyclic voltammetry and capped with HPU. The $\mathrm{I}-\mathrm{t}$ curve is collected as before, holding the potential at $+0.30 \mathrm{~V}$ to facilitate oxidation of UA. The response, represented as a calibration curve as well (Figure 4A) shows the effective selectivity provided by $\beta-\mathrm{CD}$ electropolymer results in high sensitivity $\left(1.91 \pm 0.21 \mu \mathrm{A} \cdot \mathrm{mM}^{-1}\right)$ compared to bare GCE modified with HPU only and Nafion-MWCNT/HPU (Figure 1). However, with the increase in sensitivity, the linear range of the sensor is compromised. Whereas the observed linear range for the UA concentrations using the Nafion-MWCNT/HPU composite film easily spanned the normal physiological range of 0.2 to $0.5 \mathrm{mM}$, the linear range for $\mathrm{GCE} / \beta-\mathrm{CD} / \mathrm{HPU}$ electrochemical sensor was limited to only $0.40 \mathrm{mM}$. 


\section{4. $\beta-C D / N a f i o n-M W C N T$ - Synergetic Effect}

With the efficient demonstration of the performance of the individual materials (i.e., $\beta-$ CD and Nafion-MWCNT nanocomposite) that notably enhanced UA oxidation, it follows to assess the synergistic effect of their combination. To achieve this modification, electrodes modified with Nafion-MWCNT nanocomposite films were subsequently modified via $\beta-C D$ electropolymerization as previously described. TEM and SEM imaging and IR spectroscopy suggest the materials are successfully combined. SEM imaging $\beta-C D / N a f i o n-M W C N T$ composite film shows a distinct change in the morphology of bulk material dispersed on the surface before and after $\beta-\mathrm{CD}$ electropolymerization (Fig. 2f vs. Fig. 2d, black arrows). TEM reinforces the characterization of the composite material, showing MWCNT with material modifying its walls as well as aggregates of $\beta-C D$ (Fig. 2c vs. Fig. 2e). Importantly, IR spectroscopy suggests the presence of $\beta-C D$ in the combined material as well, with the top spectra of Figure 3 exhibiting signature absorbance values of Nafion, MWCNT, and $\beta-\mathrm{CD}$. More specifically, peaks observed at 3300, 2930 and $1020 \mathrm{~cm}^{-1}$ in $\beta-C D / N a f i o n-M W C N T$ spectra corresponded to the bending vibration of $-\mathrm{OH}$ group, stretching vibrations of $-\mathrm{CH}_{2}-$ and $-\mathrm{C}-\mathrm{O}-$ $\mathrm{C}-$ groups of $\beta-\mathrm{CD}$, respectively [32]. The presence of these peaks in $\beta-\mathrm{CD} / \mathrm{Nafion}-\mathrm{MWCNT}$ films spectra suggests successful electropolymerization of $\beta-\mathrm{CD}$ on Nafion-MWCNT nanocomposite modified GCE.

Figure 5A shows the typical chronoamperometric $\mathrm{I}-\mathrm{t}$ and calibration curves of the combination of $\beta-C D$ and Nafion-MWCNT nanocomposite modified GCE towards the oxidation of UA. The sensitivity of using the combination of both materials resulted in higher $\left(2.11 \pm 0.29 \mu \mathrm{A} \cdot \mathrm{mM}^{-1}\right)$ than electrodes modified with individual materials featuring solely $\beta-\mathrm{CD}$

or Nafion-MWCNT nanocomposite while restoring the linear range of the response across a wider and clinically relevant concentration range. For a more direct comparison, Figure 5B summarizes the I-t responses of all electrodes modified with both materials simultaneously and with the individual materials. 
In order to help confirm that the $\beta-\mathrm{CD}$ is providing enhancement by sequestering UA into specific size cavities, a series of Nafion-MWCNT modified electrodes was investigated incorporating $\alpha, \beta$ and $\gamma-\mathrm{CD}$ structures. Electrode modification with Nafion-MWCNT films was performed as previously described for the electropolymerization of $\alpha, \beta$ and $\gamma-C D$ at different interfaces. The current response obtained from I-t curves during the successive injections of UA was translated to the calibration curves (Figure SI-8) and their slopes (i.e., sensitivities) compared. Figure 5C shows the greatest sensitivity was achieved using $\beta-\mathrm{CD}, \sim 3.5 \mathrm{X}$ or $\sim 2.5 \mathrm{X}$ greater than $\alpha$ and $\gamma-C D$, respectively. These results suggest that the size of the $\beta-C D$ is indeed critical for selective detection of UA.

While the $\beta-C D$ can provide effective selectivity, the degree of polymerization can also hamper sensitivity. The number of electropolymerization cycles directly affect film thickness [17], which in turn increase capacitive current (i.e., background) and depress sensitivity of the electrochemical sensing scheme. UA sensitivity as the function of the number of cycles of $\beta-\mathrm{CD}$ electropolymerization was evaluated and is illustrated in Figure SI-7 for both GCE/NafionMWCNT/ $\beta-\mathrm{CD} / \mathrm{HPU}$ and GCE/ $\beta-\mathrm{CD} / \mathrm{HPU}$ electrochemical sensors. For both sets of films, the highest sensitivity toward UA oxidation was obtained for the electrochemical sensor made using 10 cycles of the $\beta-\mathrm{CD}$ electropolymerization process, a result consistent with literature reports [17].

Taken collectively, these results suggest that the observed enhancement can be attributed to a combination of increasing the electroactive surface area with MWCNT which (a) promote fast electron transfer to the electrode interface via effective electronic coupling and (b) act as an accommodating platform for $\beta-\mathrm{CD}$ polymerization, a material that subsequently provides selectivity by bringing UA into the film for more efficient oxidation - both of which act to increase UA sensitivity.

\subsection{Uric Acid Sensor Performance Evaluation}


The composite films featuring the synergetic combination of Nafion-MWCNT with electropolymerized $\beta-\mathrm{CD}$ exhibit high sensitivity, fast response time, and dynamic/linear ranges that easily span physiologically relevant concentrations of UA. As part of evaluating the selectivity of the system, the influences of possible interfering species on the detection of UA was also investigated. The assessment of the selectivity of GCE/Nafion-MWCNT/ $\beta-\mathrm{CD} / \mathrm{HPU}$ electrochemical sensor is shown in Figure 6A where the complete film is subjected to injections of common interferents as well as UA injections of different concentrations with the electrode poised at $+0.3 \mathrm{~V}$. Injections of most interfering species (e.g., sodium nitrite, oxalic acid, and glucose) did not result in significant current response relative to the observed UA response. Ascorbic acid gave a minimal response, largely insignificant compared to that of UA. As in prior reports, the most problematic interferent for UA sensing remains acetaminophen. While this represents a challenge, it is important to remember that acetaminophen is a synthetic pharmaceutical that is ingested and clears the body in a relatively short time (4-6 hours). In any eventual medical application of these sensors, the presence of acetaminophen will be identifiable with an effective patient history $[13,57]$.

One of the advantages of the electrocatalytic activity provided by the incorporation of MWCNT is that the sensor can operate at lower potentials. The sensor presented using an applied potential of $+0.3 \mathrm{~V}$ versus many traditional sensors that use higher voltages. A prime example of such sensors is the widely explored $1^{\text {st }}$ generation schemes that detect hydrogen peroxide oxidation at $+0.65 \mathrm{~V}$ as it is a by-product of an enzymatic reaction that indirectly signals the presence of an analyte [13, 57, 58]. Figure 6A compares the current responses for common interferents at the sensor scheme developed here held at both +0.3 and $+0.65 \mathrm{~V}$. We note that some interferents (e.g., acetaminophen and sodium nitrite) showed larger amperometric responses at the electrodes held at $+0.65 \mathrm{~V}$. Additionally, after exposure to interferents, the sensor maintained sensitivity to UA concentration with successive injections of UA at $100 \mu \mathrm{M}$ and a proportional response to a $300 \mu \mathrm{M}$ increase. From the I-t responses toward interferents versus the UA analyte, selectivity coefficients $\left(\mathrm{K}^{\mathrm{amp}}\right)$ were calculated to conservatively and quantitatively assess selectivity as has been done in previous studies [14, 20, 21, 45] using the following equation: 


$$
K_{j}^{a m p}=\log \left(\frac{\Delta I_{j} / C_{j}}{\Delta I_{U A} / C_{U A}}\right)
$$

where $\Delta \mathrm{I}_{\mathrm{j}}$ and $\Delta \mathrm{I}_{\mathrm{UA}}$ are the measured currents for a specific interferent species $(\mathrm{j})$ and uric acid and $\mathrm{C}_{\mathrm{j}}$ and $\mathrm{C}_{\mathrm{UA}}$ are concentrations of the interferent species and uric acid, respectively. Negative selectivity coefficients indicate that the interferent is inconsequential whereas species with positive values are selected for by the sensor, in this case, only acetaminophen and uric acid. Figure 6A (inset), displays a graphical comparison of selectivity coefficients for the GCE/Nafion-MWCNT/ $\beta-$ CD/HPU electrochemical sensor at both +0.65 and $+0.3 \mathrm{~V}$. Figure 6A underscores the critical selectivity of the sensor with selectivity coefficients of 0.55 and 0.53 for UA compared with just 0.05 and -2.18 for sodium nitrite at +0.65 and $+0.3 \mathrm{~V}$ respectively. Negative selectivity coefficients were observed for AA, OA, and glucose. Except for AP, selectivity coefficient values for this UA electrochemical sensing are in agreement with selectivity deemed effective for other reported glucose biosensing schemes [45]. As with many other UA sensors [1], AP, with a selectivity coefficient of 0.37 and 0.33 at +0.65 and $+0.3 \mathrm{~V}$ respectively, remains a problematic species during sensing, although its selectivity coefficient reported here is lower than that reported in literature [20, 21, 58]. The electrochemical sensor exhibits an excellent response time $\left(t_{\mathrm{R}-95 \%}\right)$ of $\sim 5$ seconds, a conservative estimate of the response where the time is measured once $95 \%$ of the total current change is achieved [20, 21]. A complete table summary of the performance of the complete system and comparison of these properties to other related literature reports of uric acid sensors is provided in the supplementary materials (Table SI-2). The sensitivity and response time are generally stable for at least 15 days (Figure 6B), indicating the potential application of the developed sensor for monitoring of near term pregnant women with PIH.

\section{Conclusion}

Fabrication of a layer-by-layer UA electrochemical sensor was reported based on electropolymerization of $\beta-\mathrm{CD}$ on Nafion-MWCNT nanocomposite film modified GCE. The fabricated GCE/Nafion-MWCNT/ $\beta-\mathrm{CD} / \mathrm{HPU}$ UA electrochemical sensor showed numerous advantages such as high sensitivity, fast current response, wide dynamic range and stability. The 
simple preparation, low cost of used materials, and enhanced electrocatalytic activity of the electrochemical sensor at lower overpotential $(+0.3 \mathrm{~V})$ can open avenue for production of an applicable UA sensor.

\section{Acknowledgments}

This research was generously supported by funding from the National Science Foundation (CHE-1401593), Commonwealth Health Research Board, the College of Arts and Sciences (MAS), and the Department of Chemistry's Puryear-Topham-Pierce Endowment. We would like to specifically thank Microscopy Director Christie Davis (TEM and SEM) and Dr. Matthew Trawick (AFM) for their important contributions to this work. We gratefully acknowledge the following people for making research possible at the University of Richmond: Drs. T. Leopold, R. Kanters, D. Kellogg, R. Miller, and R. Coppage, as well as Russ Collins, Phil Joseph, Mandy Mallory, and Lamont Cheatham.

\section{References}

[1] Erden PE, Kılıç E. A review of enzymatic uric acid biosensors based on amperometric detection. Talanta 107 (2013) 312-23.

[2] Li Y, Zhai X, Wang H, Liu X, Guo L, Ji X, Wang L, Qiu H, Liu X. Non-enzymatic sensing of uric acid using a carbon nanotube ionic-liquid paste electrode modified with poly $(\beta$ cyclodextrin). Microchim. Acta 182 (2015) 1877-84.

[3] Wu S, Wang T, Gao Z, Xu H, Zhou B, Wang C. Selective detection of uric acid in the presence of ascorbic acid at physiological $\mathrm{pH}$ by using a $\beta$-cyclodextrin modified copolymer of sulfanilic acid and N-acetylaniline. Biosens. Bioelectron. 23 (2008) 1776-80.

[4] Chen X, Wu G, Cai Z, Oyama M, Chen X. Advances in enzyme-free electrochemical sensors for hydrogen peroxide, glucose, and uric acid. Microchim. Acta 181 (2014) 689-705.

[5] Chauhan N, Pundir CS. An amperometric uric acid biosensor based on multiwalled carbon nanotube-gold nanoparticle composite. Anal. Biochem. 413 (2011) 97-103.

[6] Retna Raj C, Ohsaka T. Voltammetric detection of uric acid in the presence of ascorbic acid at a gold electrode modified with a self-assembled monolayer of heteroaromatic thiol. Journal of Electroanal. Chem. 540 (2003) 69-77.

[7] Lakshmi D, Whitcombe MJ, Davis F, Sharma PS, Prasad BB. Electrochemical Detection of Uric Acid in Mixed and Clinical Samples: Rev. Electroanal. 23 (2011) 305-20.

[8] Moraes ML, Rodrigues Filho UP, Oliveira ON, Ferreira M. Immobilization of uricase in layer-by-layer films used in amperometric biosensors for uric acid. J. Solid State Electrochem. 11 (2007) 1489-95.

[9] Wang Z, Wang Y, Luo G. A selective voltammetric method for uric acid detection at [small beta]-cyclodextrin modified electrode incorporating carbon nanotubes. Analyst 127 (2002) 1353-8. 
[10] Erden PE, Kaçar C, Öztürk F, Kılıç E. Amperometric uric acid biosensor based on poly(vinylferrocene)-gelatin-carboxylated multiwalled carbon nanotube modified glassy carbon electrode. Talanta 134 (2015) 488-95.

[11] Li Y, Ran G, Yi WJ, Luo HQ, Li NB. A glassy carbon electrode modified with graphene and poly(acridine red) for sensing uric acid. Microchim. Acta 178 (2012) 115-21.

[12] Roberts JM, Bodnar LM, Lain KY, Hubel CA, Markovic N, Ness RB, Powers RW. Uric acid is as important as proteinuria in identifying fetal risk in women with gestational hypertension. Hypertension 46 (2005) 1263-9.

[13] Conway GE, Lambertson RH, Schwarzmann MA, Pannell MJ, Kerins HW, Rubenstein KJ, Dattelbaum JD, Leopold MC. Layer-by-layer design and optimization of xerogel-based amperometric first generation biosensors for uric acid. J. Electroanal. Chem. 775 (2016) 135-45.

[14] Poulos NG, Hall JR, Leopold MC. Functional Layer-By-Layer Design of Xerogel-Based First-Generation Amperometric Glucose Biosensors. Langmuir 31 (2015) 1547-55.

[15] DiPasquale LT, Poulos NG, Hall JR, Minocha A, Bui TA, Leopold MC. Structure-function relationships affecting the sensing mechanism of monolayer-protected cluster doped xerogel amperometric glucose biosensors. J. Colloid Interface Sci. 450 (2015) 202-12.

[16] Wang J. Carbon-nanotube based electrochemical biosensors: Rev. Electroanal. 17 (2005) 7 14.

[17] Alarcón-Ángeles G, Guix M, Silva WC, Ramírez-Silva MT, Palomar-Pardavé M, RomeroRomo M, Merkoçi A. Enzyme entrapment by $\beta$-cyclodextrin electropolymerization onto a carbon nanotubes-modified screen-printed electrode. Biosens. Bioelectron. 26 (2010) 176873.

[18] Hrapovic S, Liu Y, Male KB, Luong JHT. Electrochemical Biosensing Platforms Using Platinum Nanoparticles and Carbon Nanotubes. Anal. Chem. 76 (2004) 1083-8.

[19] Baghayeri M, Amiri A, Farhadi S. Development of non-enzymatic glucose sensor based on efficient loading Ag nanoparticles on functionalized carbon nanotubes. Sens. Actuators B: Chem. 225 (2016) 354-62.

[20] Freeman MH, Hall JR, Leopold MC. Monolayer-Protected Nanoparticle Doped Xerogels as Functional Components of Amperometric Glucose Biosensors. Anal. Chem. 85 (2013) 4057-65.

[21] Wayu MB, Pannell MJ, Leopold MC. Layered Xerogel Films Incorporating MonolayerProtected Cluster Networks on Platinum-Black-Modified Electrodes for Enhanced Sensitivity in First-Generation Uric Acid Biosensing. ChemElectroChem 3 (2016) 12451252.

[22] Du J, Yue R, Ren F, Yao Z, Jiang F, Yang P, Du Y. Novel graphene flowers modified carbon fibers for simultaneous determination of ascorbic acid, dopamine and uric acid. Biosens. Bioelectron. 53 (2014) 220-4.

[23] Wang C, Du J, Wang H, Zou Ce, Jiang F, Yang P, Du Y. A facile electrochemical sensor based on reduced graphene oxide and Au nanoplates modified glassy carbon electrode for simultaneous detection of ascorbic acid, dopamine and uric acid. Sens. Actuators B: Chem. 204 (2014) 302-9.

[24] Schnorr JM, Swager TM. Emerging Applications of Carbon Nanotubes. Chem. Mater. 23 (2011) 646-57. 
[25] Shi J, Claussen JC, McLamore ES, ul Haque A, Jaroch D, Diggs AR, Calvo-Marzal P, Rickus JL, Porterfield DM. A comparative study of enzyme immobilization strategies for multi-walled carbon nanotube glucose biosensors. Nanotechnol. 22 (2011) 355502.

[26] Gooding JJ. Nanostructuring electrodes with carbon nanotubes: A review on electrochemistry and applications for sensing. Electrochim. Acta 50 (2005) 3049-60.

[27] Wang J, Musameh M, Lin Y. Solubilization of Carbon Nanotubes by Nafion toward the Preparation of Amperometric Biosensors. J. ACS.125 (2003) 2408-9.

[28] Yang H, Zhu Y, Chen D, Li C, Chen S, Ge Z. Electrochemical biosensing platforms using poly-cyclodextrin and carbon nanotube composite. Biosens. Bioelectron. 26 (2010) 295-8.

[29] Wayu MB, King JE, Johnson JA, Chusuei CC. A Zinc Oxide Carbon Nanotube Based Sensor for In Situ Monitoring of Hydrogen Peroxide in Swimming Pools. Electroanal. 27 (2015) 2552-8.

[30] Li J, Feng H, Feng Y, Liu J, Liu Y, Jiang J, Qian D. A glassy carbon electrode modified with $\beta$-cyclodextin, multiwalled carbon nanotubes and graphene oxide for sensitive determination of 1,3-dinitrobenzene. Microchim. Acta 181 (2014) 1369-77.

[31] Gao Y-S, Wu L-P, Zhang K-X, Xu J-K, Lu L-M, Zhu X-F, Wu Y. Electroanalytical method for determination of shikonin based on the enhancement effect of cyclodextrin functionalized carbon nanotubes. Chin. Chem. Lett. 26 (2015) 613-8.

[32] He Y, Xu Z, Yang Q, Wu F, Liang L. Supramolecular modification of multi-walled carbon nanotubes with $\beta$-cyclodextrin for better dispersibility. J. Nanopart. Res. 17 (2015) 1-10.

[33] Ni J-A, Ju H-X, Chen H-Y, Leech D. Amperometric determination of epinephrine with an osmium complex and Nafion double-layer membrane modified electrode. Anal. Chim. Acta 378 (1999) 151-7.

[34] Liaw H-W, Chen J-M, Tsai Y-C. Development of an Amperometric Ethanol Biosensor Based on a Multiwalled Carbon Nanotube-Nafion-Alcohol Dehydrogenase Nanobiocomposite. J. Nanosci. Nanotechnol. 6 (2006) 2396-402.

[35] Burmeister JJ, Pomerleau F, Palmer M, Day BK, Huettl P, Gerhardt GA. Improved ceramicbased multisite microelectrode for rapid measurements of 1-glutamate in the CNS. J. Neurosci. Methods 119 (2002) 163-71.

[36] Lin Y, Lu F, Tu Y, Ren Z. Glucose Biosensors Based on Carbon Nanotube Nanoelectrode Ensembles. Nano Lett. 4 (2004) 191-5.

[37] Tsai Y-C, Li S-C, Chen J-M. Cast Thin Film Biosensor Design Based on a Nafion Backbone, a Multiwalled Carbon Nanotube Conduit, and a Glucose Oxidase Function. Langmuir 21 (2005) 3653-8.

[38] Xu Q, Liu N-N, Zhu J-J. Lead Determination on MWNT/Nafion Composite Modified Glassy Carbon Electrodes. Chin. J. Chem. 23 (2005) 1510-4.

[39] Tian L, Zhang B, Sun D, Chen R, Wang B, Li T. A thin poly(acridine orange) film containing reduced graphene oxide for voltammetric simultaneous sensing of ascorbic acid and uric acid. Microchim. Acta 181 (2014) 589-95.

[40] Rafati AA, Afraz A, Hajian A, Assari P. Simultaneous determination of ascorbic acid, dopamine, and uric acid using a carbon paste electrode modified with multiwalled carbon nanotubes, ionic liquid, and palladium nanoparticles. Microchim. Acta 181 (2014) 19992008.

[41] Nau WM, Florea M, Assaf KI. Deep Inside Cucurbiturils: Physical Properties and Volumes of their Inner Cavity Determine the Hydrophobic Driving Force for Host-Guest Complexation. Isr. J. Chem. 51 (2011) 559-77. 
[42] Szejtli J. Introduction and General Overview of Cyclodextrin Chemistry. Chem. Rev. 98 (1998) 1743-54.

[43] Soylemez S, Hacioglu SO, Kesik M, Unay H, Cirpan A, Toppare L. A Novel and Effective Surface Design: Conducting Polymer/beta-Cyclodextrin Host-Guest System for Cholesterol Biosensor. Acs Appl. Mater. Interfaces 6 (2014) 18290-300.

[44] Zheng L, Wu S, Lin X, Nie L, Rui L. Selective Determination of Uric Acid by Using a $\beta$ Cyclodextrin Modified Electrode. Electroanal. 13 (2001) 1351-4.

[45] Koh A, Lu Y, Schoenfisch MH. Fabrication of Nitric Oxide-Releasing Porous Polyurethane Membranes-Coated Needle-type Implantable Glucose Biosensors. Anal. Chem. 85 (2013) 10488-94.

[46] Bott A, Heineman W. Chronocoulometry. Curr Sep. 20 (2004) 121-6.

[47] Karyakin AA, Karyakina EE, Schmidt H-L. Electropolymerized Azines: A New Group of Electroactive Polymers. Electroanal. 11 (1999) 149-55.

[48] Finklea H. Electrochemistry of organized monolayers of thiols and related molecules on electrodes. Electroanal. Chem. Series Adv. 19 (1996) 109-335.

[49] Fang B, Zhang C, Zhang W, Wang G. A novel hydrazine electrochemical sensor based on a carbon nanotube-wired $\mathrm{ZnO}$ nanoflower-modified electrode. Electrochim. Acta 55 (2009) $178-82$.

[50] Peigney A, Laurent C, Flahaut E, Bacsa RR, Rousset A. Specific surface area of carbon nanotubes and bundles of carbon nanotubes. Carbon 39 (2001) 507-14.

[51] Saito T, Matsushige K, Tanaka K. Chemical treatment and modification of multi-walled carbon nanotubes. Physica B: Condens. Matter 323 (2002) 280-3.

[52] He Y, Fu P, Shen X, Gao H. Cyclodextrin-based aggregates and characterization by microscopy. Micron 39 (2008) 495-516.

[53] Liu Y-L, Su Y-H, Chang C-M, Suryani, Wang D-M, Lai J-Y. Preparation and applications of Nafion-functionalized multiwalled carbon nanotubes for proton exchange membrane fuel cells. J. Mater. Chem. 20 (2010) 4409-16.

[54] Li C, Sun G, Ren S, Liu J, Wang Q, Wu Z, Sun H, Jin W. Casting Nafion-sulfonated organosilica nano-composite membranes used in direct methanol fuel cells. J. Membr. Sci. 272 (2006) 50-7.

[55] Liang Z, Chen W, Liu J, Wang S, Zhou Z, Li W, Sun G, Xin Q. FT-IR study of the microstructure of Nafion ${ }^{\circledR}$ membrane. J. Membr. Sci. 233 (2004) 39-44.

[56] Sahoo NG, Bao H, Pan Y, Pal M, Kakran M, Cheng HKF, Li L, Tan LP. Functionalized carbon nanomaterials as nanocarriers for loading and delivery of a poorly water-soluble anticancer drug: a comparative study. Chem. Commun. 47 (2011) 5235-7.

[57] Chen K, Conway GE, Hamilton GA, Trawick ML, Leopold MC. Electropolymerized layers as selective membranes in first generation uric acid biosensors. J. Appl. Electrochem. 46 (2016) 1-13.

[58] Koh A, Riccio DA, Sun B, Carpenter AW, Nichols SP, Schoenfisch MH. Fabrication of nitric oxide-releasing polyurethane glucose sensor membranes. Biosens. Bioelectron. 28 (2011) 17-24. 
CNT-Project.VER10 


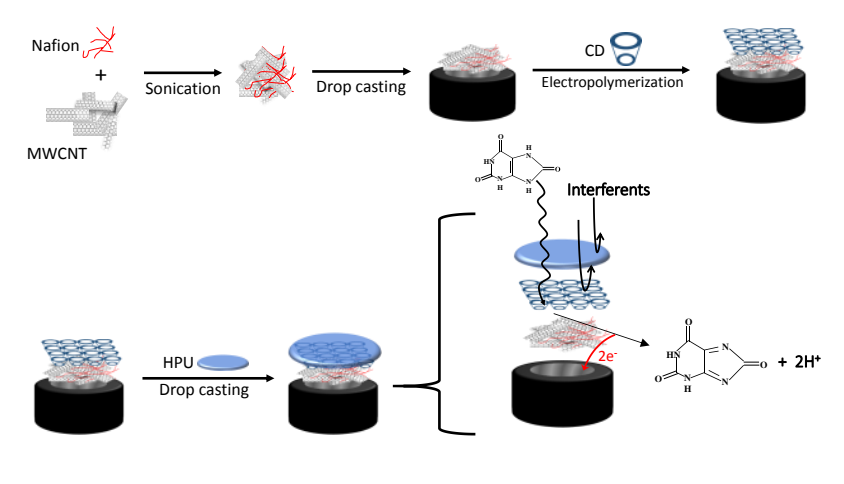

Scheme 1: Schematic representation of the LbL UA electrochemical sensor

nical sensor



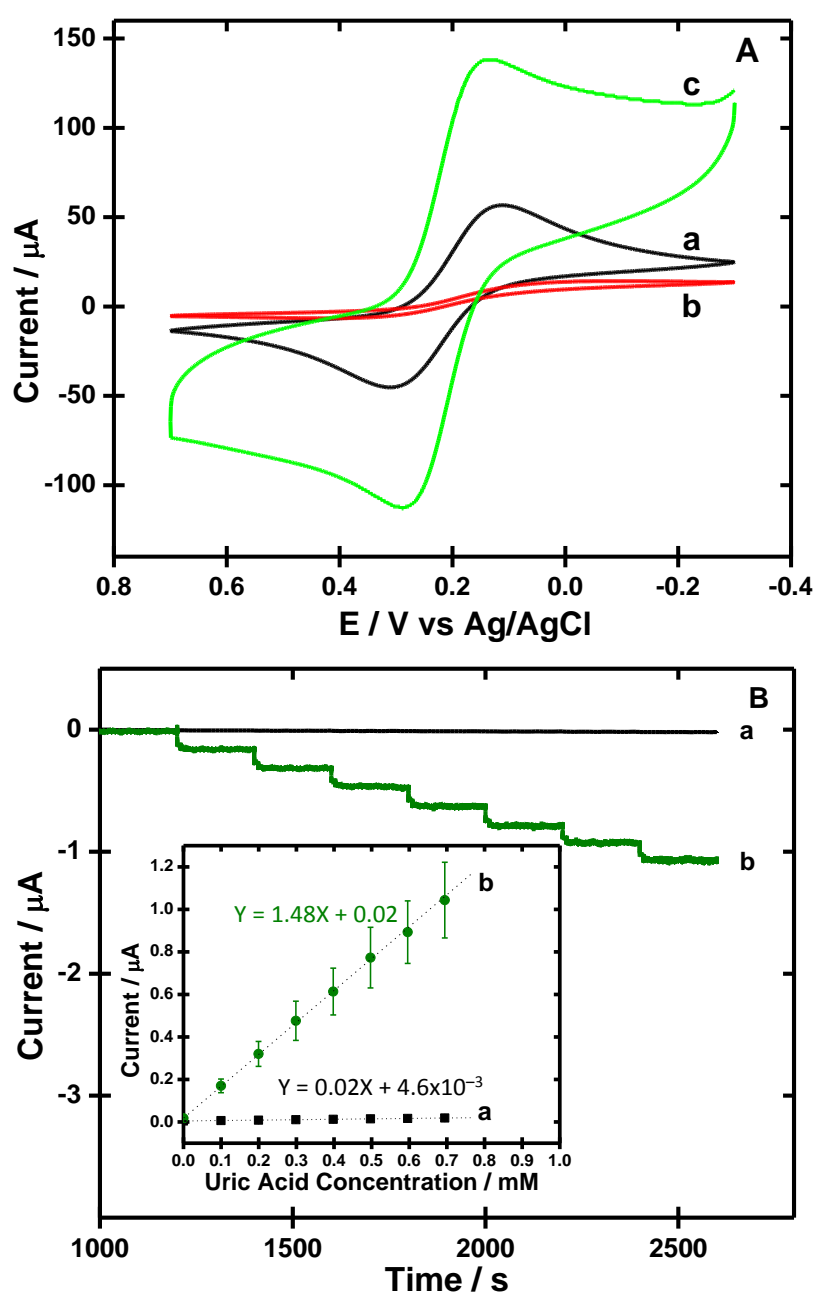

Figure 1. (A) Cyclic voltammetry of $5 \mathrm{mM}$ potassium ferricyanide $(0.5 \mathrm{M} \mathrm{KCl})$ at (a) bare GCE, (b) Nafion modified GCE and (c) Nafion-MWCNT nanocomposite films modified GCE; (B) Representative amperometric I-t curves (obtained at $+0.3 \mathrm{~V}$ vs $\mathrm{Ag} / \mathrm{AgCl}$ ) and corresponding calibration curves (inset) during successive $0.1 \mathrm{mM}$ injections of uric acid at (a) bare and (b) Nafion-MWCNT composite film modified GCEs with HPU capping layer immersed in 65.55 mM PBS ( $\mathrm{pH} 7.0$ ). Note: In some cases, standard error bars are smaller than markers for average value $(n=6)$. 

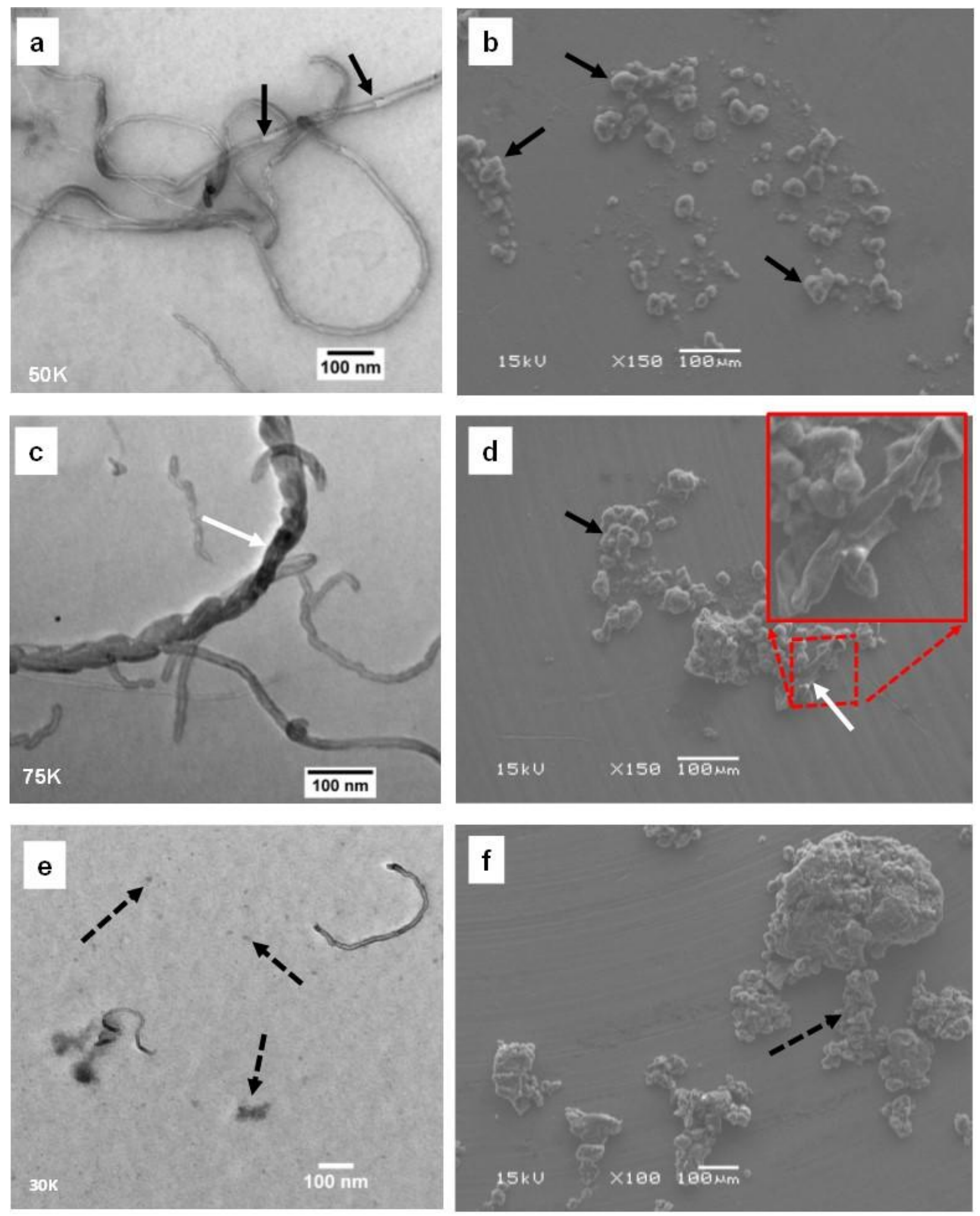

Figure 2: TEM (left) and SEM (right) images of pristine MWCNT (a,b), Nafion-MWCNT $(\mathbf{c}, \mathbf{d})$ and Nafion-MWCNT/b-CD (e,f) respectively. 


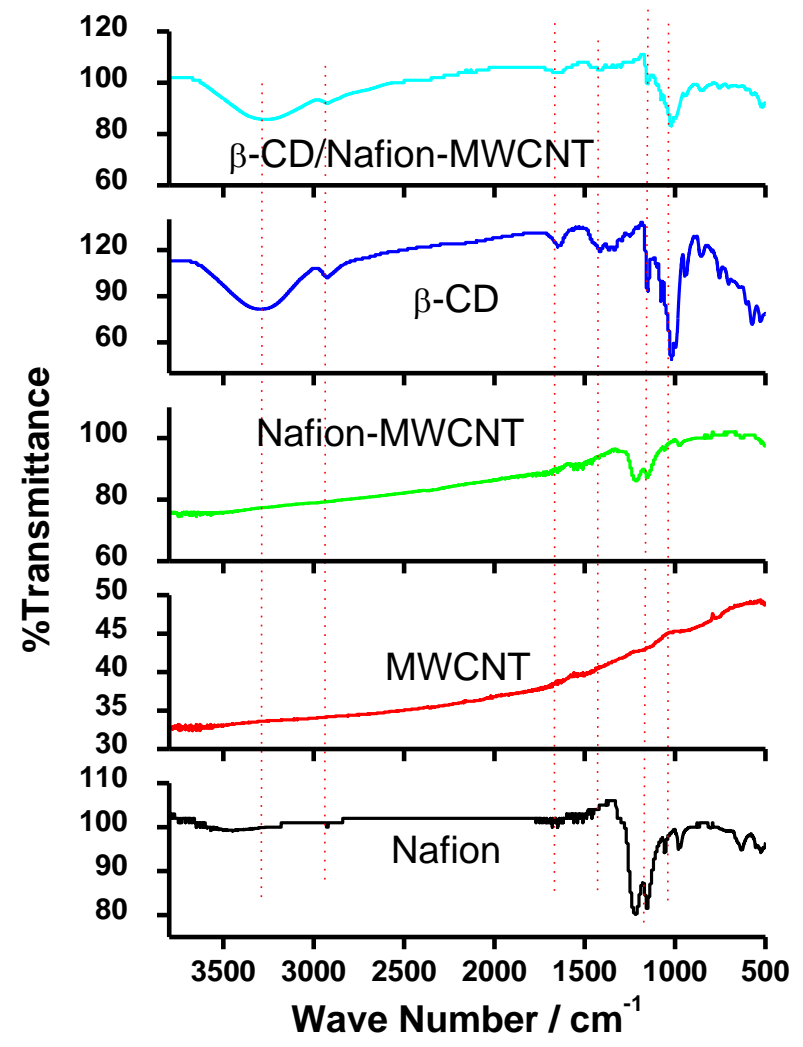

Figure 3: Characterization of various electrode materials by FTIR spectroscopy. 


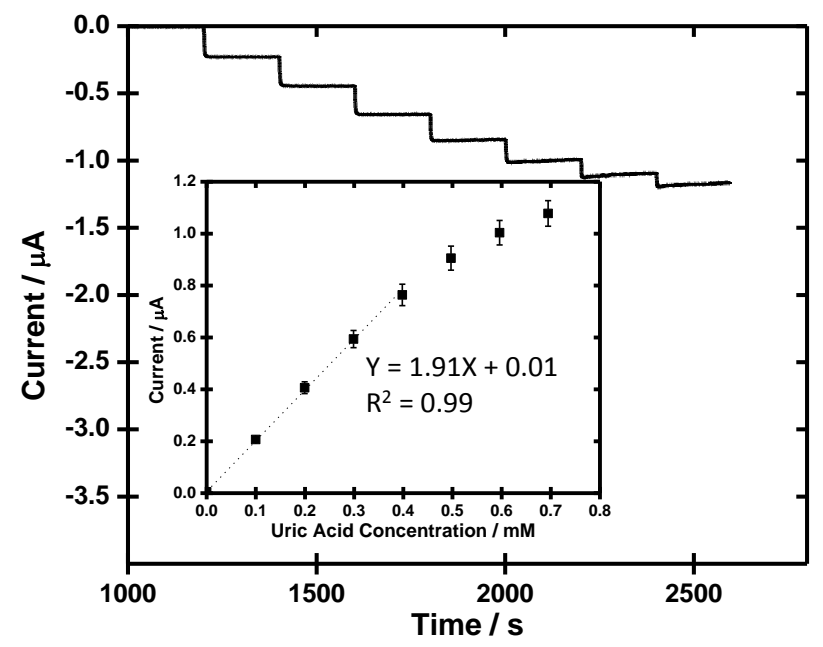

Figure 4: Representative amperometric I-t curve and corresponding calibration curve (inset) during successive $0.1 \mathrm{mM}$ injections of uric acid at glassy carbon electrode modified with $\beta$ $\mathrm{CD}$, coated with HPU, and immersed in $65.55 \mathrm{mM}$ PBS (pH 7.0). Note: In some cases, standard error bars are smaller than markers for average value $(n=6)$. 

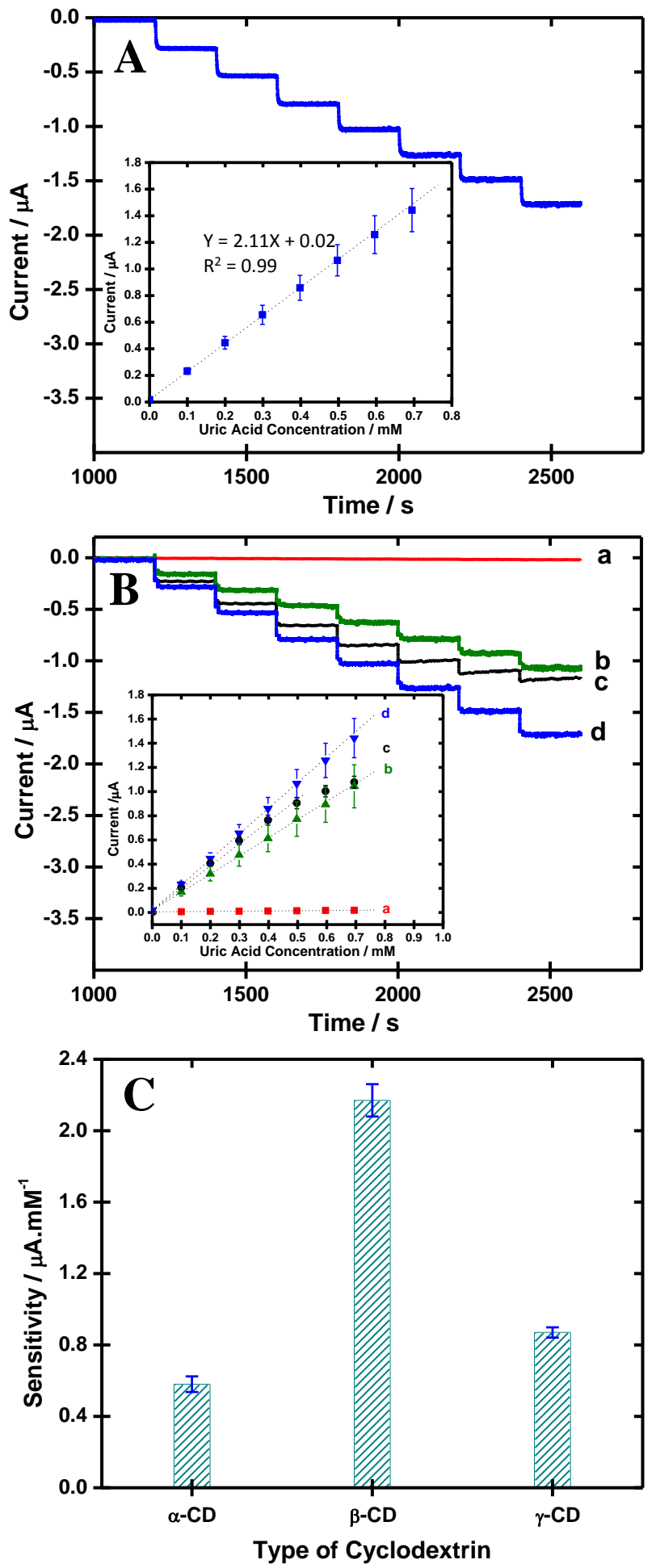

Figure 5: (A) Representative amperometric I-t curve and corresponding calibration curve (inset) during successive $0.1 \mathrm{mM}$ injections of uric acid at glassy carbon electrode modified with bCD/Nafion-MWCNT and then coated with HPU. (B) Representative amperometric I-t curves and corresponding calibration curves (inset) during successive $0.1 \mathrm{mM}$ injections of uric acid at glassy carbon electrodes; bare (a) and modified with Nafion-MWCNT (b), $\beta-\mathrm{CD}(\mathbf{c})$ and $\beta-\mathrm{CD} /$ Nafion-MWCNT (d); each coated with HPU. (C) Sensitivity of GCE/Nafion-MWCNT/ $\alpha-$ $\mathrm{CD} / \mathrm{HPU}, \quad \mathrm{GCE} / \mathrm{Nafion}-\mathrm{MWCNT} / \beta-\mathrm{CD} / \mathrm{HPU}$ and GCE/Nafion-MWCNT/ $\gamma-\mathrm{CD} / \mathrm{HPU}$ electrochemical sensor towards the oxidation of UA . Solution conditions: $65.55 \mathrm{mM}$ PBS $(\mathrm{pH}$ 7.0).

Note: In some cases, standard error bars are smaller than markers for average value $(n=6)$. 

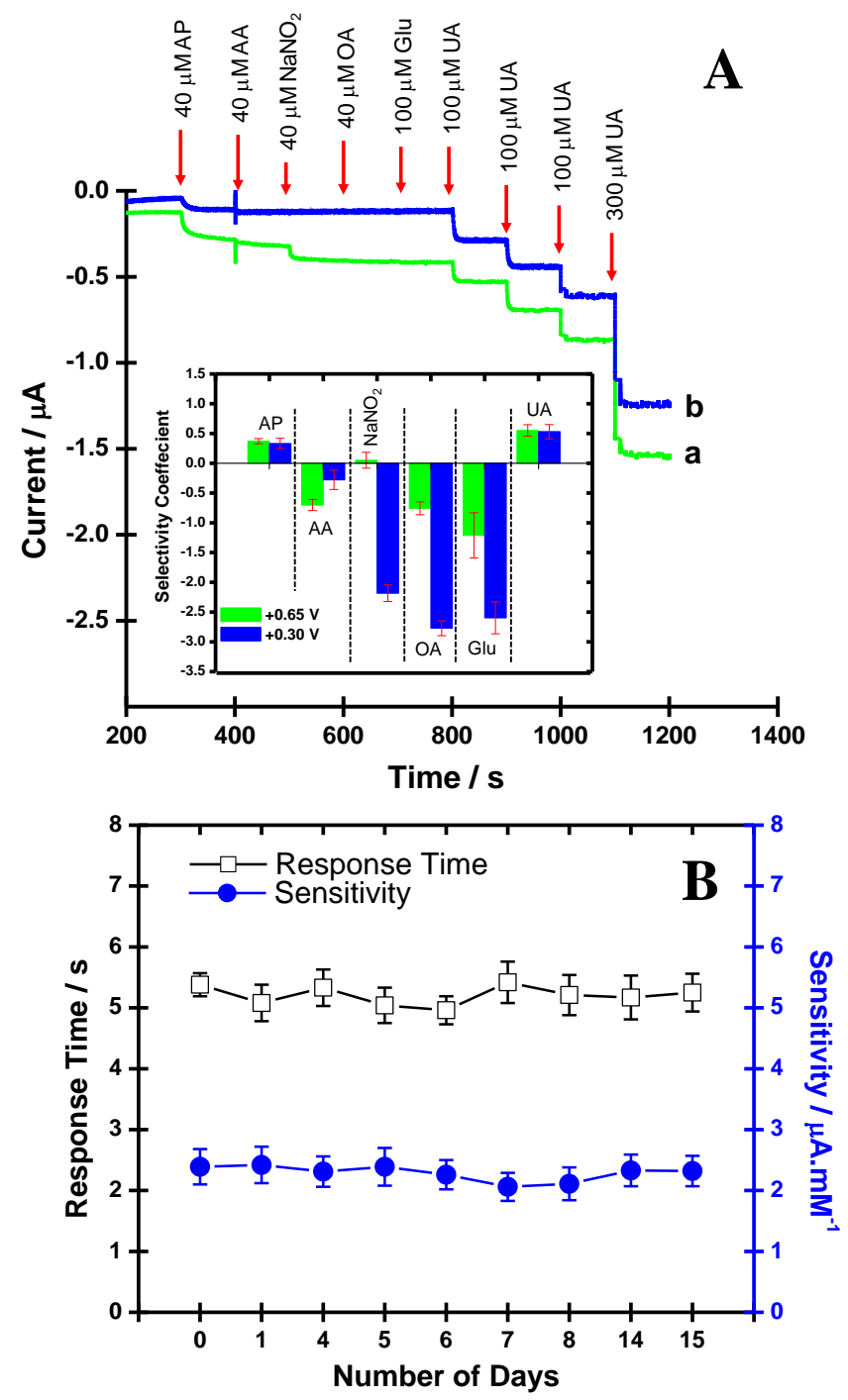

Figure 6: (A) Typical amperometric I-t curves of GCE/Nafion-MWCNT/ $\beta-C D / H P U$ electrochemical sensor during injections of common interferent species and UA and a graphical summary (inset) of selectivity coefficients for acetaminophen (AP), ascorbic acid (AA), $\mathrm{NaNO}_{2}$, oxalic acid (OA), and glucose (Glu) at a) +0.65 and b) $+0.30 \mathrm{~V}$ vs $\mathrm{Ag} / \mathrm{AgCl}$. (B) Response time $\left(\mathrm{t}_{\mathrm{R}-}\right.$ $95 \%$ and sensitivity of GCE/Nafion-MWCNT/ $\beta-\mathrm{CD} / \mathrm{HPU}$ UA electrochemical sensor over a period of 8 days. Note: In some cases, standard error are smaller than markers for average value $(n=8)$. 